\title{
Abcès tuberculeux de la paroi thoracique chez une patiente immunocompétente au centre hospitalier universitaire de Dakar (Sénégal)
}

\section{Tuberculosis Abscess of the Chest Wall on an Immunocompetent Patient in the Teaching Hospital of Dakar (Senegal)}

\author{
A. Massaly $\cdot$ L.A. Ndiaye $\cdot$ V.M.P. Cissé Diallo $\cdot$ B. Wembulua $\cdot$ N.A. Lakhe $\cdot$ K. Diallo Mbaye $\cdot$ D. Ka $\cdot$ A. Dièye $\cdot$ \\ M. Diop · N.M. Fall · A.S. Badiane $\cdot$ D. Thioub $\cdot$ L. Fortes Déguénonvo $\cdot$ C.T. Ndour $\cdot$ M. Seydi
}

Reçu le 7 mai 2018 ; accepté le 31 juillet 2018

(C) Société de pathologie exotique et Lavoisier SAS 2018

Résumé La tuberculose demeure un problème de santé publique, malgré les nombreuses avancées significatives notées dans le diagnostic ces dernières années. L'atteinte de la paroi thoracique est une forme extrapulmonaire rare. Sa présentation clinique est non spécifique, et une tuméfaction thoracique collectée en est la principale manifestation clinique. Le diagnostic est amélioré par l'utilisation d'outils non invasifs tels que le test Xpert MTB/Rif ${ }^{\circledR}$ avec une bonne sensibilité sur le pus. La prise en charge repose sur le traitement médical quelquefois associé à un traitement chirurgical.

Mots clés Tuberculose $\cdot$ Paroi thoracique $\cdot$ Xpert MTB/Rif ${ }^{\circledR}$. Hôpital · Dakar · Sénégal · Afrique intertropicale

\begin{abstract}
Tuberculosis remains a problem of public health, in spite of the numerous significant advances noted in the diagnosis in recent years. The involvement of the thoracic wall is a rare extra lung shape. Its clinical presentation is not specific and a collected thoracic tumefaction is the main clinical demonstration. The diagnosis is improved by the use of non-invasive tools such as the Xpert MTB/Rif ${ }^{\circledR}$ test with a good sensibility on the pus. Medical care is based on medical treatment and sometimes associated with surgical treatment.
\end{abstract}

A. Massaly $(\bowtie) \cdot$ L.A. Ndiaye $\cdot$ V.M.P. Cissé Diallo

B. Wembulua $\cdot$ N.A. Lakhe $\cdot$ K. Diallo Mbaye $\cdot$ D. Ka $\cdot$

A. Dièye $\cdot$ N.M. Fall $\cdot$ A.S. Badiane $\cdot$ D. Thioub

L. Fortes Déguénonvo · C.T. Ndour · M. Seydi

Service des maladies infectieuses Ibrahima-Diop-Mar

centre hospitalier universitaire de Dakar, Sénégal

e-mail : massalyaminata@gmail.com

V.M.P. Cissé Diallo $\cdot$ N.A. Lakhe $\cdot$ K. Diallo Mbaye $\cdot$ D. Ka

L. Fortes Déguénonvo · C.T. Ndour · M. Seydi

Service des maladies infectieuses,

faculté de médecine et pharmacie,

université Cheikh-Anta-Diop, Dakar, Sénégal
Keywords Tuberculosis $\cdot$ Thoracic wall $\cdot$ Xpert MTB/Rif ${ }^{\circledR}$. Hospital $\cdot$ Dakar $\cdot$ Senegal $\cdot$ Sub-Saharan Africa

\section{Introduction}

La tuberculose est une maladie endémique qui constitue un véritable problème de santé publique en Afrique subsaharienne [13]. La localisation extrapulmonaire est de plus en plus fréquente et représente 20 à $40 \%$ des cas de tuberculose rapportés selon certaines études [3,8,21], et l'atteinte musculosquelettique en représente 1 à $5 \%[13,16,20]$. Le diagnostic de ces formes est souvent difficile, et l'on a parfois recours à des examens invasifs tels que la biopsie avec examen anatomopathologique pour une confirmation. Cependant, l'utilisation du test Xpert MTB/Rif ${ }^{\circledR}$, fondé sur la détection moléculaire de l'ADN de Mycobacterium tuberculosis, et la sensibilité à la rifampicine ont considérablement révolutionné et facilité le diagnostic de la tuberculose. L'OMS a approuvé l'utilisation de ce test depuis décembre 2010 et le recommande comme test diagnostique initial en cas de suspicion de tuberculose résistante ou de tuberculose associée au VIH, et depuis octobre 2013 pour les tuberculoses extrapulmonaires [12,14].

Nous rapportons un cas de tuberculose de la paroi thoracique chez une jeune femme de 24 ans, dont le diagnostic a été établi grâce au test de Xpert MTB/ Rif $^{\circledR}$ sur le liquide de ponction de la masse thoracique.

\section{Observation}

Il s'agit d'une jeune femme âgée de 24 ans, étudiante, sans notion de contage tuberculeux ni antécédents pathologiques rapportés. L'interrogatoire retrouvait un tableau clinique 
évoluant depuis plus d'un an, marqué par l'apparition successive de masses sus-claviculaires, puis axillaires bilatérales douloureuses. L'évolution s'était faite vers la fistulisation avec issue de pus franc, puis vers la cicatrisation au bout de deux mois. Après plusieurs consultations avec une notion d'antibiothérapie à base d'amoxicilline-acide clavulanique et de cotrimoxazole sans succès, elle consulte un an après le début de la symptomatologie au service des maladies infectieuses de Fann. Parallèlement est apparue une tuméfaction sus-mammaire droite indolore. Par ailleurs, la patiente rapportait une fièvre modérée, intermittente, un amaigrissement progressif non chiffré, une asthénie physique et une anorexie non sélective. L'examen physique retrouvait une patiente amaigrie, avec des muqueuses conjonctivales pâles, une température à $37,5^{\circ} \mathrm{C}$, un poids de $40 \mathrm{~kg}$ avec un IMC à $18 \mathrm{~kg} / \mathrm{m}^{2}$. L'examen de la paroi thoracique retrouvait une tuméfaction sus-mammaire droite, ovoïde, d'environ $8 \mathrm{~cm}$ de diamètre, indolore, de consistance molle, mobile par rapport au plan profond et superficielle avec une peau en regard d'aspect normal (Fig. 1). Au niveau axillaire gauche, on notait la présence d'adénopathies de 1 à $2 \mathrm{~cm}$ de diamètre non inflammatoires, à droite, de multiples cicatrices atrophiques donnant un aspect d'écrouelles (Figs 1, 2). L'examen pleuropulmonaire et des autres appareils était normal.

Sur le plan paraclinique, l'hémogramme montrait une anémie à 10,6 g/dl normochrome normocytaire et une hyperleucocytose à $11000 / \mathrm{mm}^{3}$ à polynucléaires neutrophiles. L'échographie de la masse thoracique retrouvait une collection liquidienne échogène mesurant $104 \times 22 \mathrm{~mm}$ de grand axe et semblant se développer aux dépens du muscle thora-

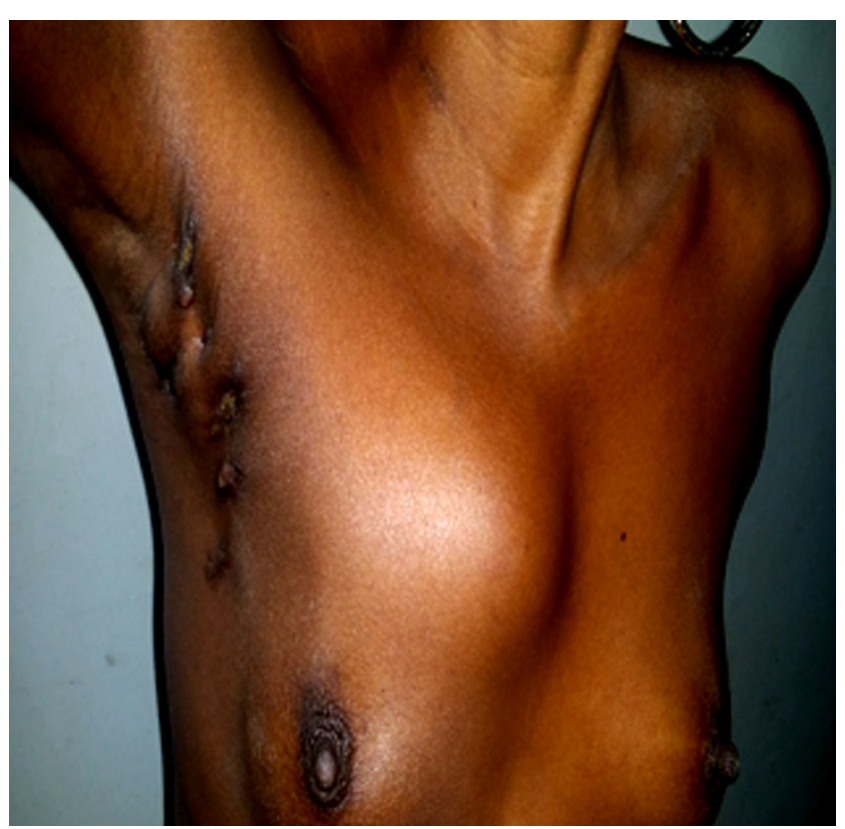

Fig. 1 Tuméfaction sus-mammaire droite / Tumefaction above the right breast

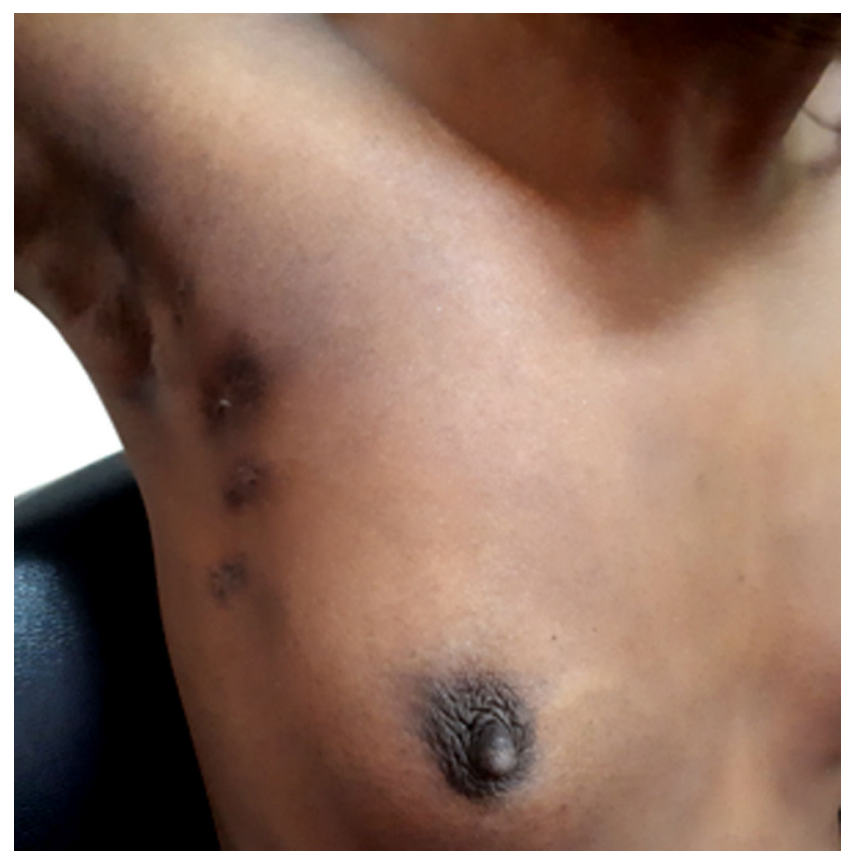

Fig. 2 Évolution clinique à huit mois de traitement / Clinical evolution after 8 months of treatment

cique antérieur et infiltrant le tissu glandulaire. La tomodensitométrie thoracique montrait de multiples collections hypodenses à rehaussement annulaire, développées aux dépens des muscles pectoraux, dont la plus volumineuse à droite mesurait $82 \times 24 \mathrm{~mm}$ de diamètre. Celles-ci étaient associées à des adénopathies axillaires bilatérales et à une ostéolyse avec sclérose bordante de la corticale en T2 (Figs 3, 4). Il n'y avait pas de lésions pleuroparenchymateuses.

La ponction de la collection avait ramené du pus franc. La recherche de BAAR était revenue négative, et le test Xpert MTB/Rif ${ }^{\circledR}$ sur le pus était positif à M. tuberculosis sans résistance à la rifampicine. La sérologie rétrovirale était négative. Ainsi, le diagnostic d'une tuberculose multifocale de la paroi thoracique et ganglionnaire a été retenu. La patiente a été mise sous traitement antituberculeux pendant huit mois, avec une bonne évolution clinique (Fig. 2).

\section{Discussion}

La tuberculose peut atteindre tous les organes avec un polymorphisme clinique. Sa localisation musculosquelettique surtout de la paroi thoracique reste rare et inhabituelle. Elle représente $0,1 \%$ de l'ensemble des cas de tuberculose et 1 à $5 \%$ des localisations musculosquelettiques [20]. Cette atteinte survient selon trois mécanismes : il peut s'agir d'une extension directe à partir d'un foyer pleuropulmonaire, d'une atteinte par voie lymphatique à partir d'adénopathies ou d'une dissémination par voie hématogène [23]. Chez 
notre patiente, l'atteinte ganglionnaire homolatérale, précédant l'apparition de la masse thoracique, suggère un mécanisme par dissémination lymphatique. Du point de vue physiopathologique, le bacille tuberculeux atteint les chaînes lymphatiques internes et intercostales. Ces adénopathies se

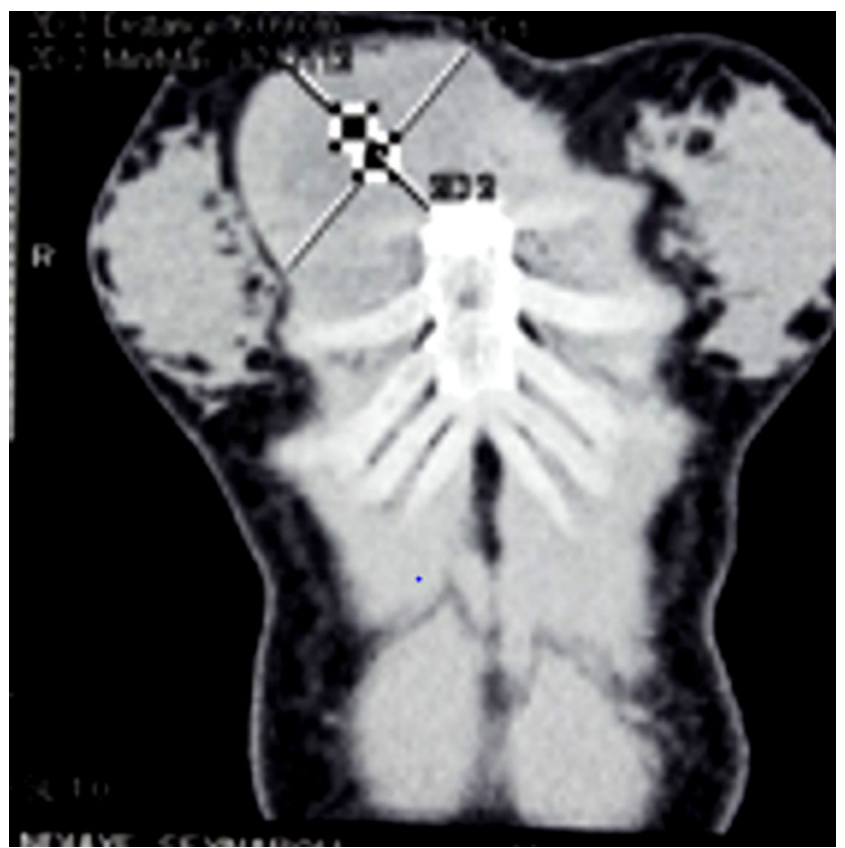

Fig. 3 Scanner thoracique montrant une volumineuse collection développée aux dépens des muscles pectoraux / Chest CT scan showing a large collection developed at the expense of the pectoral muscles

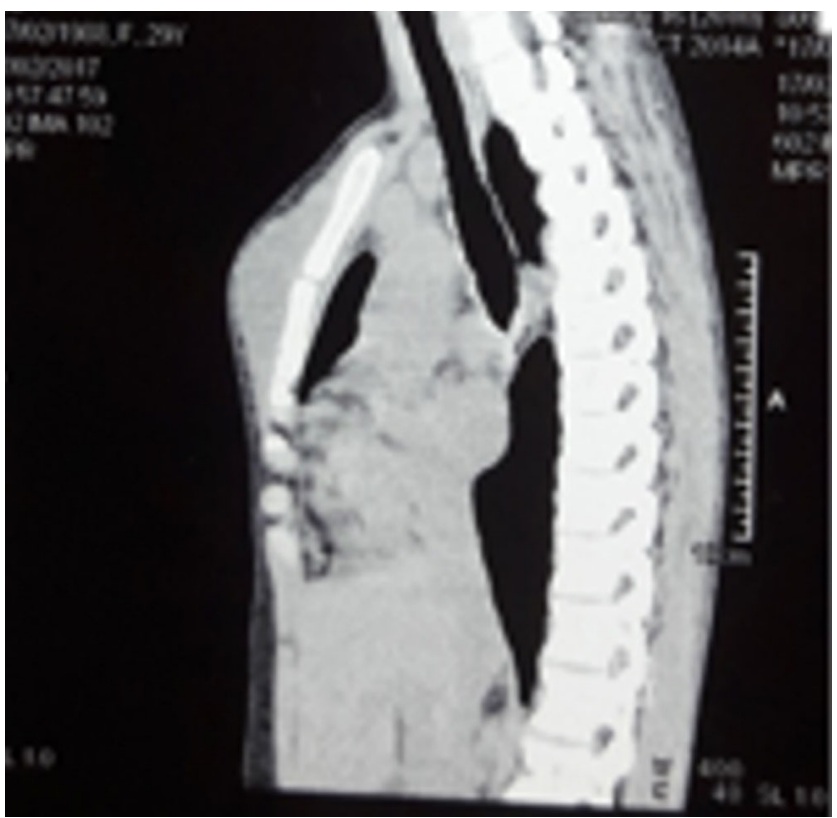

Fig. 4 Collection parasternale droite / Parasternal right collection ramollissent avec formation d'un caséum qui va se rompre et s'étendre dans les tissus de voisinage pour aboutir à la formation d'un abcès froid.

Les manifestations cliniques sont non spécifiques. La durée d'évolution des symptômes est variable, allant d'un mois à un an selon les séries $[5,7,23]$. Ce délai était d'un an chez notre patiente, ce qui rend compte du diagnostic tardif de la tuberculose extrapulmonaire dans notre contexte. L'apparition d'une tuméfaction pariétale thoracique est la principale circonstance de découverte. Elle était présente dans $100 \%$ des cas dans la série de Benjelloun et al. et dans $92 \%$ des cas dans la série de Kim et al. [2,9]. Ainsi, le diagnostic peut être évoqué dans notre contexte devant un ensemble d'arguments épidémiologiques tels que la notion de contage tuberculeux, la chronicité du tableau clinique, l'altération de l'état général. L'atteinte peut être pariétale, isolée ou associée à d'autres localisations, d'où l'intérêt de l'imagerie médicale dans le cadre du bilan d'orientation diagnostique et de la recherche des autres localisations [7]. La tomodensitométrie est l'examen idéal, car elle permet d'évaluer l'atteinte des tissus mous et son étendue, l'extension des lésions osseuses, l'atteinte pleuropulmonaire et ganglionnaire [7,11]. D'autres examens tels que la scintigraphie et l'imagerie par résonance magnétique sont plus performants pour la détection de lésions osseuses [10]. Le diagnostic de la tuberculose pariétale peut être difficile, car elle peut être confondue avec une pathologie tumorale, d'autres infections à pyogènes, à fungiques ou les autres mycobactérioses. Ainsi, la confirmation diagnostique se fait par mise en évidence du bacille tuberculeux dans les prélèvements par la bacilloscopie ou la culture sur milieu solide et liquide. L'examen anatomopathologique sur biopsie peut être parfois nécessaire pour confirmer le diagnostic en montrant un granulome épithélioïde gigantocellulaire avec nécrose caséeuse. En plus de ces techniques diagnostiques, l'avènement de la biologie moléculaire a considérablement amélioré le diagnostic de la tuberculose. La sensibilité de ces tests varie de 60 à $80 \%$ [18]. Des tests tels que le Xpert MTB/Rif ${ }^{\mathbb{B}}$ ont montré leur capacité à confirmer la tuberculose en un temps record de deux heures et de détecter la sensibilité à la rifampicine. La sensibilité et la spécificité de ce test sont variables selon les études. Sur le liquide de pus, sa sensibilité est de $92 \%[4,5,19]$. Ce test pourrait être utilisé dans le diagnostic de cette forme clinique de tuberculose et éviter ainsi le recours systématique à des techniques de diagnostic invasives telles que la biopsie.

Le traitement de la tuberculose de la paroi thoracique repose sur les antituberculeux associés ou non à un traitement chirurgical. Les molécules utilisées sont la rifampicine, l'isoniazide, l'éthambutol et la pyrazinamide. Il n'y pas de consensus sur la stratégie thérapeutique appropriée pour le traitement de cette forme clinique de tuberculose. L'association d'un traitement chirurgical et antituberculeux est la plus 
préconisée dans la littérature pour réduire les risques de récidive $[1,6,17,22]$. Certains auteurs suggèrent que le traitement médical seul est efficace [9], comme ce fut le cas pour notre patiente : nous n'avons pas observé de récidive à un an. La durée du traitement antituberculeux est variable selon les séries. La durée moyenne est de six mois, mais pouvant aller jusqu'à huit, voire 12 mois.

\section{Conclusion}

La tuberculose de la paroi thoracique est une entité rarement rapportée dans la littérature. Elle entre souvent dans le cadre d'une tuberculose multifocale. Elle peut être confondue avec une masse d'origine tumorale ou une autre infection. Le diagnostic de cette forme de tuberculose n'est pas souvent aisé, compte tenu de la nature paucibacillaire du produit pathologique. Le test Xpert MTB/Rif ${ }^{\mathbb{R}}$, du fait de sa sensibilité et de sa spécificité sur le pus, est un examen d'un grand apport dans le diagnostic rapide de cette forme de tuberculose.

Liens d'intérêts : Les auteurs déclarent ne pas avoir de liens d'intérêts.

\section{Références}

1. Aghajanzadeh M, Pourrasouli Z, Aghajanzadeh G, et al (2010) Surgical treatment of chest wall tuberculosis. Tanaffos 93:28-32

2. Benjelloun H, Morad S, Zaghba N, et al (2015) Les abcès froids pariétaux thoraciques chez les sujets immunocompétents. Pan Afr Med J 20:161. doi: 10.11604/pamj.2015.20.161.5773

3. Burrill J, Williams CJ, Bain G, et al (2007) Tuberculosis: a radiologic review. Radiographics 27:1255-73

4. Diallo AB, Kollo AI, Camara M, et al (2016) Performance du GeneXpert MTB/Rif ${ }^{\circledR}$ dans le diagnostic de la tuberculose extrapulmonaire à Dakar : 2010-2015. Pan Afr Med J 25:129. doi: 10.11604/pamj.2016.25.129.10065

5. El Barni R, Lahkim M, Achour A (2013) Abcès tuberculeux de la paroi thoracique chez l'enfant. Pan Afr Med J 14:9. doi: 10.11604/pamj.2013.14.9.2138

6. Gayathri Devi DR, Narayanaswamy YV, Areena H (2012) Recurrent cold abscess of the chest wall in a young immunocompetent person. Indian Landrecies 2(1): 26-8

7. Grover SB, Jain M, Dumeer S, et al (2011) Chest wall tuberculosis. A clinical and imaging experience. Indian J Radiol Imaging 21:8-33. doi: 10.4103/0971-3026.76051
8. Gunal S, Yang Z, Agarwal M, et al (2011) Demographic and microbial characteristics of extrapulmonary tuberculosis cases diagnosed in Malatya, Turkey, 2001-2007. BMC Public Health 11:154. doi: 10.1186/1471-2458-11-154

9. Kim YT, Han KN, Kang CH, et al (2008) Complete resection is mandatory for tubercular cold abscess of the chest wall. Ann Thorac Surg 85:273-7

10. Leonard MK, Blumberg HM (2017) Musculoskeletal tuberculosis. Microbiol Spectr 5(2):1-21. TNMI7-0046-2017.

11. Morris BS, Maheshwari M, Chalwa A (2004) Chest wall tuberculosis: a review of CT appearances. Br J Radiol 77:449-57

12. OMS (2013) Technique automatisée d'amplification de l'acide nucléique en temps réel pour la détection rapide et simultanée de la tuberculose et de la résistance à la rifampicine : utilisation du test Xpert MTB/Rif ${ }^{\circledR}$ pour le diagnostic de la tuberculose pulmonaire et de la tuberculose extrapulmonaire chez l'adulte et chez l'enfant : mise à jour de la politique, $86 \mathrm{p}$

13. OMS (2014) Global tuberculosis report 2014, $171 \mathrm{p}$

14. OMS (2011) Mise en œuvre rapide du test diagnostique Xpert $\mathrm{MTB} / \mathrm{Rif}^{(\mathbb{R}}$ : guide technique et opérationnel — considérations pratiques, $39 \mathrm{p}$

15. Ozkutuk N, Surucüoglu S (2014) Evaluation of the Xpert MTB/ Rif $^{(B)}$ assay for the diagnosis of pulmonary and extrapulmonary tuberculosis in an intermediate-prevalence setting. [Article en turque] Mikrobiyol Bul 48:223-32

16. Peto HM, Pratt RH, Harrington TA, et al (2009) Epidemiology of extrapulmonary tuberculosis in the United States, 1993-2006. Clin Infect Dis 49:1350-7. doi: 10.1086/605559

17. Tanaka S, Aoki M, Nakanishi T, et al (2012) Retrospective case series analysing the clinical data and treatment options of patients with a tubercular abscess of the chest wall. Interact Cardiovasc Thorac Surg 14:249-52. doi: 10.1093/icvts/ivr113

18. Tiwari V, Jain A, Verma RK (2003) Application of enzyme amplified mycobacterial DNA detection in the diagnosis of pulmonary and extrapulmonary tuberculosis. Indian J Med Res 118:224-8

19. Vadwai V, Boehme C, Nabeta P, et al (2011) Xpert MTB/Rif ${ }^{\mathbb{R}}$ : a new pillar in diagnosis of extrapulmonary tuberculosis? J Clin Microbiol 49:2540-5. doi: 10.1128/JCM.02319-10.

20. Wang X, Yang Z, Fu Y, et al (2014) Insight to the epidemiology and risk factors of extrapulmonary tuberculosis in Tianjin, China during 2006-2011. PLoS One 9:e112213. doi: 10.1371/journal. pone. 0112213

21. Yone EW, Kengne AP, Moifo B, Kuaban C (2013) Prevalence and determinants of extrapulmonary involvement in patients with pulmonary tuberculosis in a Sub-Saharan African country: a cross-sectional study. Scand J Infect Dis 45:104-11. doi: 10.3109/00365548.2012.714905

22. Zhang L, Han C, Han Z, et al (2016) Two rare cases involving the spread of tuberculosis: a tuberculous abscess of the chest wall invading the liver by way of the diaphragm. Intern Med 55:22379. doi: 10.2169/internalmedicine.55.5692

23. Zidane A, Bakzaza O, Afandi O, et al (2015) Tuberculose de la paroi thoracique : à propos de trois cas. Rev Pneumol Clin 71:290-3. doi: 10.1016/j.pneumo.2014.12.006 